\title{
Clima social escolar y el autoconcepto en estudiantes en una institución educativa nacional de Lima Norte
}

\author{
School social climate and self in a national students in college of North Lima \\ Jorge Alberto Flores Morales ${ }^{1}$ \\ Lidia Neyra Huamaní
}

\section{RESUMEN}

El objetivo de la investigación fue determinar la correlación entre la calidad del clima social escolar y el autoconcepto. El tipo de investigación fue basica de nivel descriptivo cuyo diseño de investigación fue correlacional y de enfoque cuantitativo. La población censal estuvo conformada por 164 estudiantes de colegio nacional cuyas edades fluctuaban entre 16 y 18 años. La técnica que se utilizó fue la encuesta. El instrumento de recolección de datos fue el cuestionario para evaluar el clima social del centro (CECSE) construido a partir de los items del California School Climate and Safety Survey adaptado por Trianes, et al (2006). Además, el cuestionario de autoconcepto GARLEY de Belen Garcia Torres. Para la confiabilidad de ambos instrumentos se utilizó el alfa de Cronbach cuyos resultados fueron: 0.73 para la variable clima social escolar y 0.77 para la variable autoconcepto. En la presente investigación se arribó a la conclusión: existe relación entre el clima social escolar y autoconcepto obteniéndose un coeficiente de correlación positiva de rs= $0.495^{* *}, p$ valor $=0.000<0.05$ en los estudiantes del 4 to y 5 to de secundaria de la Institución. Asimismo, la percepción en cuanto al autoconcepto de los estudiantes varones y mujeres no hay diferencia ya que puntúan un nivel de tendencia alto. De igual forma, en lo referente al clima escolar consideran el ambiente de manera positiva.

\section{Palabras clave}

Clima social, autoconcepto, autoestima, adolescentes, violencia escolar.

\begin{abstract}
The goal of the research was to determine the correlation between the quality of the school social climate and the self-concept. This research was basic, of descriptive level, correlational design and quantitative approach. The census population comprised a total of 164 students from a public school, with ages between 16 and 18 years old. The technique used was the survey. The instrument to collect data was the questionnaire to evaluate the social climate in the institution (CECSE), created from the items of California School Climate and Safety Survey adapted by Trianes, et al (2006). Besides, the questionnaire of self-concept GARLEY de Belén García Torres. For the reliability of both instruments, it was needed Cronbach's alpha, which results were: 0.73 for the school social climate variable and 0.77 for the self-concept variable.

In this research the conclusion was that there is a connection between the school social climate and the self-concept, showing a positive correlational coefficient $r s=0.495^{* *}, p$ value $=0.000<0.05$ in students of eleventh to twelfth secondary grade in the institution. Besides, there is no difference in the perception of self-concept of male and female students, since they both have a high level. Likewise, in the case of school climate, they consider the environment as positive.
\end{abstract}

\section{Keywords}

Social climate, self-concept, self-esteem, adolescents, school violence.

\footnotetext{
Doctor y Magister en Administración de la educación, Licenciado en Psicología y Educación, Bachiller en Teología. Docente de Pre y Postgrado.

2 Doctora en Ciencias de la Educación, Magister en Didáctica de la Comunicación, Licenciada en Lengua y Literatura y Bachiller en Educación. Docente de Pre y Pos Grado.
} 


\section{INTRODUCCIÓN}

En muchos casos el comportamiento de los estudiantes en el aula se expresa con manifestaciones de violencia, bajo rendimiento, acoso escolar, etc. Con frecuencia se ve influido por la percepción de un clima social escolar adverso o permisivo que da cobijo a esas actitudes. Esa situación se agrava si el estudiante ha desarrollado un autoconcepto disminuido de sí mismo. Lo cual, genera mayor índice de insatisfacción en el ambiente y en su persona. Entonces, evaluar el clima social permite al sistema escolar objetivar y tomar contacto con la realidad en la que viven los estudiantes. Al mismo tiempo, indagar sobre su percepción de sí mismo en los distintos ámbitos donde desarrolla su experiencia de aprendizaje (Sánchez, 2009).

En el ambiente educativo, el clima social escolar y el autoconcepto son generadores de distintos comportamientos en términos de promover o bloquear conductas prosociales y construir percepciones negativas o positivas acerca de sí mismos y del ambiente que les rodea. Por ello mismo, los gestores educativos han de promover un ambiente escolar positivo donde el estudiante tenga la posibilidad de desarrollar un autoconcepto alto de sí que fomente una actitud de apertura hacia la comunidad y favorezca talentos personales $e$ iniciativas creativas en mejora de su contexto (Estela, 2013).

Esta situación negativa que se expresa a nivel nacional, nos lleva a reflexionar sobre la problemática de la convivencia de los estudiantes en sus centros educativos, en especial de los últimos años de estudio en Educación Básica Regular. Además, sobre cómo se asocia con el autoconcepto personal del estudiante en las diversas facetas de su existencia. Así, cuando se percibe un clima escolar adverso, el núcleo íntimo de la persona esta se ve afectada.

Lamentablemente, nuestras instituciones educativas solo priorizan las acciones académicas curriculares y desatienden el bienestar emocional de los alumnos. Ello incide, con el tiempo, en efectos negativos de convivencia dentro del aula y en creencias falsas sobre las cualidades personales (Peralta y Sánchez, 2006).

Esa situación problemática de la calidad del clima social escolar se asocia a que cada uno de los estudiantes se sienta mal o bien dentro de ella. Si es percibida como un ambiente agresivo y violento de hecho que puede impactar de forma negativa en la autoimagen del estudiante en diversas facetas de su vida personal. En este contexto, es necesario explorar el autoconcepto que tienen los estudiantes de sí mismos y la percepción de su centro educativo. Si les resulta seguro o no al estar en contacto con sus compañeros de clase, además de evaluar el trato de los docentes hacia ellos (Valdez y Carlos, 2014).

\subsection{Revisión de la Literatura}

Fariña, García y Vilariño (2010) comprobaron que los factores psicosociales y biológicos actúan en la adolescencia como elementos de riesgo o de protección ante el comportamiento antisocial y delictivo. Entre sus conclusiones más relevantes señalan que los efectos intersujetos ponen de manifiesto que los menores de reforma tienen menos desarrollado en autoconcepto emocional y familiar que los normalizados.

Por otro lado, Meza (2010) señaló que el hábito de estudio es necesario si se quiere progresar en el campo educativo. Asimismo, que conviene sacar el máximo provecho con técnicas adecuadas. En este caso, la perseverancia, en cuanto rasgo de personalidad, ayuda a obtener buenos resultados.

Asimismo, Chuquillanqui (2012), en su estudio sobre el funcionamiento familiar $y$ autoconcepto de los alumnos del sexto grado de las instituciones educativas de la RED 8 Callao, evidenció que entre las dos dimensiones estudiadas en el funcionamiento familiar, es la cohesión la que estaría determinando los niveles adecuados del desarrollo del autoconcepto de los niños.

De acuerdo a Epstein (1990), el autoconcepto es una realidad compleja que está integrada por 
diversos autoconceptos como el físico, social, emocional y académico. Se caracteriza como una realidad concreta y dinámica generada a través de la experiencia, especialmente social, donde las personas significativas son la clave para la comprensión de los pensamientos, sentimientos y conductas o comportamientos de las personas.

De igual forma, Arón y Milic (1999) expresaron que el clima social escolar es el escenario donde se desarrolla las condiciones ambientales de interacción entre directivos, docentes, alumnos y padres de familia y la percepción que tienen estos sobre la escuela.

\section{MATERIAL}

\subsection{Clima social}

El clima social en el aula es un conjunto de situaciones percibidas tanto por los docentes y alumnos que tienen características idiosincráticas propias que lo distingue de los demás ambientes (Moos, 1979). La base de esta propuesta está en las percepciones que los miembros del grupo tienen de las interacciones dentro del aula. Ella es dada por las interrelaciones alumno-alumno y profesoralumno.

Moos (1979) agrupa el clima del aula en seis tipos que se manifiestan y estructuran de la siguiente manera en aulas orientadas a:

\section{La innovación}

La relación estructurada

El rendimiento académico con apoyo del profesor

La colaboración solidaria

La competición individual desmesurada

El control.

Este autor desarrolla una escala de clima social escolar a partir de los estudios realizados en la Universidad de Standford, California, en su laboratorio de Ecología Social (Moos, R.; Moos, B. y Tricket, 1989). Estos tres últimos autores proponen las siguientes dimensiones:

Relaciones: incluye los aspectos de la interacción profesor-alumno y alumno- alumno. Esta interacción se evidencia en las clases, debates y otras actividades académicas.

Autorrealización: orientación a metas, se refieren al funcionamiento especifico del ambiente de clase. Se expresa en la importancia de cumplir con las tareas encomendadas y en la valoración del esfuerzo personal.

Estabilidad y Cambio: se relaciona con las normas y reglamentos que marcan las innovaciones de la clase y el profesor. Se expresa en la importancia que se da al cumplimiento del trabajo bien ejecutado. Además, en el cumplimiento de las normas y las consecuencias que se derivan de no acatarlas como el grado de seguridad con que el profesor aplica las normas, etc.

Asimismo, Arón y Milic (1999) señalaron que el clima social escolar es el escenario donde se desarrollan las condiciones ambientales de interacción entre directivos, docentes, alumnos y padres de familia. Incluso la percepción que los individuos tienen de los distintos aspectos del ambiente en el cual desarrollan sus actividades habituales, en este caso el colegio. Después de un tiempo, Arón y Milic (2000) añadieron que existen climas sociales escolares tóxicos y nutritivos. Es por ello que "Un clima social positivo es donde las personas son sensibles a las situaciones difíciles y son capaces de dar apoyo emocional" (p. 118). Las características de un clima social nutritivo o positivo son:

Se percibe un clima de justicia

Reconocimiento explícito de los logros

Predomina la valoración positiva

Tolerancia a los errores

Sensación de ser alguien valioso

Sentido de pertenencia

Conocimiento de las normas y consecuencias de sus transgresiones

Flexibilidad de las normas

Sentirse respetado en su dignidad, en su individualidad, en sus diferencias

Acceso y disponibilidad a información relevante

Favorece el crecimiento personal

Favorece la creatividad

Permite el enfrentamiento constructivo de conflictos. 
Asimismo, Arón y Milic (2000, p. 118) definen que los climas sociales tóxicos son "aquellos que contaminan el ambiente contagiándolo con características negativas que parecieran hacer aflorar las partes más negativas de las personas".

Las características de un clima social tóxico o negativo son:

Percepción de injusticia

Ausencia de reconocimiento y o descalificación Predominio de la crítica

Sobrefocalización de los errores

Sensación de ser invisible

Sensación de marginalidad de no pertenencia

Desconocimiento y arbitrariedad de las normas y las consecuencias de su trasgresión

Rigidez de las normas

No sentirse respetado en su dignidad, en su individualidad, en sus diferencias

Falta de transparencia en los sistemas de información

Interferencia en el crecimiento personal, etc. (Arón y Milic, 2000 p. 120)

De hecho, al evocar la frase "clima social escolar positivo o clima social escolar de amigable" el consenso no es unánime. Pero indica que el alumno se siente cómodo o apoyado por los docentes y compañeros de clase. De esta forma, percibe al centro educativo como un buen ambiente donde desarrolla su experiencia estudiantil.

Entonces, el clima indicado para educar es un ambiente social positivo. El cual permite la convivencia de todos sus miembros en las actividades colectivas. En este contexto se expresan valores positivos de progreso académico y emocional.

En esta misma línea, Trianes, et ál. (2006) afirmaron que el clima social escolar es la percepción favorable y positiva de los estudiantes y docentes sobre la clase. Por tanto, este da cuenta de una convivencia escolar pacífica. Obviamente, esta situación potencia el aprendizaje de los estudiantes. Es por ello, que estos autores parten del punto de vista del alumno relativo al centro de estudio y relativo al profesorado al analizar el cuestionario CECSCE.

\subsection{Autoconcepto}

En la actualidad hay confusión por el término autoconcepto. Esto se debe por la naturaleza del término y sus implicancias de su medición o evaluación del yo. Para la corriente del psicoanálisis iniciada por Freud el "yo" es la estructura que integra las fuerzas del ello y superyó dando el soporte al aparato psíquico. En este sentido, la persona mantiene el equilibro con el principio de la realidad y con el concepto de sí mismo en el mundo. Frente a esta mirada interna del sujeto, la corriente conductista entre cuyos representantes connotados están Watson, Thornike, Skinner y otros, no la toma en cuenta. Es que para el conductismo los procesos internos tales como la conciencia, el yo, la autoestima, la atención, etc. son carentes de rigor científico. En cambio, el comportamiento sí puede ser analizado experimentalmente.

A pesar que el conductismo tuvo mucha repercusión en el campo de la psicología y en la educación. El estudio del self (yo, sí-mismo) no queda de todo relegado. Pues las corrientes psicológicas de corte fenomenológico y humanista mantienen el concepto del yo como un aspecto central de su propuesta. Para Rollo May, Rogers, Maslow y Kelly el sujeto percibe el mundo de manera singular y su experiencia dentro de ella es su realidad única. El self configura su experiencia y percepciones siendo conocido simbólicamente como autoconcepto.

Apreciamos como el autoconcepto juega un papel fundamental en el desarrollo de la personalidad tal como lo toman en cuenta las corrientes psicológicas. Se busca un autoconcepto positivo que ayude al sujeto a enfrentar el mundo que lo rodea. Es por ello que en el campo educativo se busque el desarrollo socio afectivo de los estudiantes y docentes.

En este sentido, consideramos a Esnaola, Goñi y Madariaga (2008) quienes señalaron:

el logro de un equilibrio socioafectivo en el alumnado a partir de una imagen ajustada y positiva de sí mismo figure entre las finalidades tanto de la Educación Primaria 
como de la Educación Secundaria Obligatoria. De ahí también que lograr un autoconcepto positivo sea uno de los objetivos más pretendidos en numerosos programas de intervención psicológica (educativa, clínica, comunitaria, cívica...) para los que se demandan estrategias $y$ recursos que permitan su mejora. (p. 70)

Al revisar los factores asociados a la persona nos encontramos con tres acercamientos básicos comúnmente aceptados: Primero, el cognitivo relativo al pensamiento, este al analizarlo hace referencia al factor autoconcepto. Segundo, el afectivo relativo al sentimiento, corresponde al factor autoestima. Tercero, el conativo referido al comportamiento, corresponde al factor autoeficacia.

Entonces, el autoconcepto tendría que limitarse a los aspectos descriptivos o cognoscitivos del self y la autoestima para designar los aspectos evaluativos y afectivos (Loperena, 2008). Pero estas realidades en la vida humana van juntas y son difíciles de disociar, debido a que toda percepción sobre uno mismo implica una valoración afectiva porque la autoestima, como componente afectivo del autoconcepto, es uno de los factores más importantes.

En este sentido, el autoconcepto se asocia a los aspectos cognitivos del conocimiento de uno mismo. Por otro lado, la autoestima está más vinculada a los aspectos evaluativos $y$ afectivos. En resumidas cuentas, el autoconcepto es una organización cognitiva y afectiva. Además, el autoconcepto está conformado por los juicios evaluativos que los jóvenes llevan a cabo sobre sus atributos y es una construcción tanto cognitiva como social. (Harter, 1999). Así, las percepciones en las diferentes dimensiones del autoconcepto ayudan a entender el comportamiento de los sujetos. Por ejemplo, un estudiante que se percibe con alta competencia matemática, se verá reforzado con altas calificaciones en dicha área. Más aún, su conducta se abocará a mantenerlas a través del tiempo.

De igual forma, García y Musitu (2009) señalaron que la autoestima expresa el concepto que uno tiene de sí mismo según sus cualidades que son susceptibles de valoración y subjetivación proveniente de su experiencia y que la persona la considera como positivas o negativas. Entonces, el autoconcepto se presenta como producto de la actividad reflexiva del individuo sobre sí mismo. Además, involucra componentes emocionales, sociales, físicos y académicos. En ella se configuran las percepciones de sí mismo que son aceptadas por la conciencia y el conocimiento.

De acuerdo a Epstein (1990) el autoconcepto es una realidad compleja, está integrada por diversos autoconceptos como el físico, social, emocional y académico. Se caracterizan como una realidad concreta y dinámica a través de la experiencia, especialmente de las sociales, donde las personas significativas son la clave para la comprensión de los pensamientos, sentimientos y conductas o comportamientos de las personas. En cambio, la autoestima hace referencia al aprecio, estima o amor que cada individuo siente sobre sí mismo. Así, como señala Gonzales (2011 p. 33 citando a Hattie, 1992) los términos como "autovaloración, autoidentidad, autoimagen, autopercepción, autoconocimiento y autoconciencia son equiparables a autoconcepto; mientras los que mejor se intercambian con el término autoestima son los de autoconsideración, autogeneración, autoaceptación, autorrespeto, autovalía, autosentimiento y autoevaluación”.

Markus y Kunda (1986) consideran que el autoconcepto es un proceso que se construye en interacción recíproca entre el sujeto y el medio. Influye en la conducta mediado por el afecto y la motivación. En tanto que el constructo de autoconcepto operativo se hace accesible y se configura en base al contexto social, al estado motivacional y al afectivo en que se halla el sujeto; afecta los cambios temporales en la autoestima, las emociones, el pensamiento y la acción. Los cuales funcionan como un incentivo para la conducta futura, al mismo tiempo, son el contexto para evaluar $e$ interpretar la percepción de sí mismo en el presente.

Cuando el niño logra un autoconcepto positivo aceptándose como es y resaltando sus cualidades, su autoestima se va fortaleciendo. 
Por ello, los padres deben tener cuidado de lo que dicen a sus hijos en todo tiempo y, sobre todo, en la manera cómo lo dicen. Si a los niños le decimos que son inteligentes amables, capaces de asumir compromisos, etc. muy probablemente crecerán asumiendo estas palabras como ciertas que generaran actitudes positivas. (De Narvaéz, 2002). El autoconcepto se presenta como una estructura cognitiva de elementos organizados en una relación semántica que se da en esta interrelación sujeto y entorno social.

De esta manera, el autoconcepto según Branden (1993) no es un simple concepto, sino que ella misma lleva una serie de imágenes y perspectivas abstractas respecto a sus rasgos sean reales o imaginarios. Entonces, el autoconcepto resulta ser un término más amplio que el de la autoestima. La imagen sería pensar la autoestima como un círculo dentro de otro mayor correspondiente al autoconcepto. Este autor señaló, además, que la autoestima representa una evaluación de la conciencia que no se basa en éxitos o fracasos o conocimientos o habilidades debido que uno puede estar seguro de sí mismo en el nivel fundamental. Pero al sentirse inseguro en situaciones particulares del trabajo, casa, amigos, etc., de hecho, se podrá desenvolverse figurativamente en el ambiente social y ser dubitativo interiormente.

Teniendo en cuenta estos fundamentos, asumimos en este estudio el autoconcepto como un conjunto de conocimientos, actitudes y sentido que tiene cada persona hacia sí mismo, todo lo cual asigna características y atributos para describirse y conocerse. Es una apreciación descriptiva y de matiz cognitivo. Obviamente, influye en la actuación de cada persona, además, no es estático, se va formando en un proceso activo de construcción a lo largo de la vida sobre la base de las experiencias propias en relación con los demás.

\section{MÉTODO}

En el presente estudio se utilizó el método hipotético-deductivo desde un enfoque cuantitativo. El cual consiste en partir de un supuesto o afirmación por demostrar para luego llegar a descomponer en sus variables y a continuación deducir los indicadores de cada uno de ellos con la finalidad de recoger información a partir de los indicadores (Centty, 2006).

3.1 Tipo y diseño: Los tipos de investigación, según Valderrama (2012, p. 38), son básica, aplicada y tecnológica. Este estudio corresponde al tipo de investigación básica porque "busca poner aprueba una teoría con escasa o ninguna intención de aplicar sus resultados a problemas prácticos".

El Diseño correspondiente, de acuerdo con Hernández, Fernández y Baptista (2010), es no experimental, transaccional, descriptivocorrelacional. Es un diseño de estudio descriptivo porque mide y reporta el Clima social escolar y el Autoconcepto en la población seleccionada. Es un estudio correlacional porque mide el grado de relación o asociación entre las variables descritas.

3.2 Población y muestra: Valderrama (2013 p. 182) indicó que la población "es el conjunto de la totalidad de las medidas de la(s) variable(s) en estudio en cada una de las unidades del universo". En nuestro caso, la población y muestra estuvo constituida por 164 estudiantes de la Institución Educativa No. 2040 -Comas, 2013. En este caso es una población censal.

\subsection{Técnicas e Instrumentos de Recolección de datos}

La técnica es la encuesta y el instrumento son los cuestionarios de: Clima social escolar y autoconcepto.

El cuestionario para evaluar el clima social del centro (CECSCE) fue construido a partir de los ítems del California School Climate and Safety Survey. El CECSCE muestra una estructura factorial estable Oblimin con dos factores de clima social: 1) relativo al centro, y 2) relativo al profesorado. Ambos factores presentan una correlación que oscila entre 0.42 y 0.48. y explican un $54,2 \%$ y un $45,6 \%$ de la varianza, respectivamente. Este instrumento validado y adaptado a lengua española con una consistencia interna para cada uno de 
los factores un alfa de 0.77 para el factor clima referente al centro y 0.72 para el factor clima referente al profesorado según Trianes y otros (2006) de la Universidad de Málaga. En Latinoamérica en Chile lo adapta Guerra y otros (2010) que concluyen que presenta adecuados índices de fiabilidad y validez (factorial y convergente). En este estudio se obtuvo una adecuada fiabilidad, obteniéndose un alfa de 0,859 .

El segundo instrumento utilizado fue el cuestionario de autoconcepto GARLEY de Belén García Torres autora de este cuestionario con la asesoría estadística de Rosario Martínez Arias. El instrumento permite el estudio del Autoconcepto desde una perspectiva multidimensional y considera seis dimensiones: Autoconcepto Físico, Autoconcepto Social, Autoconcepto intelectual, Autoconcepto Familiar, Autoconcepto como Sensación de control y el Autoconcepto personal.

La autora trabajó con una muestra de 957 sujetos de los cuales 485 eran varones y 472 mujeres. La selección de este instrumento se debe a que es una herramienta muy útil para el estudio del autoconcepto en sujetos que pueden leer con fluidez (7-8 años) hasta el final de la escolarización (17-18 años). La fiabilidad de la prueba se ha obtenido mediante el coeficiente de Cronbach y arroja un valor de 0.87 que se puede considerar indicativo de alta consistencia interna.

\section{RESULTADOS}

Tabla 1: Variable clima social y autoconcepto

\section{Correlaciones}

\begin{tabular}{lllcc}
\hline \hline & & clima_social & autoconcepto \\
\hline \multirow{4}{*}{ Rho de Spearman } & Coeficiente de correlación & 1.000 & $0.495^{* *}$ \\
& & Sig. (bilateral) &. & 0.000 \\
& & N & 164 & 164 \\
& \multirow{2}{*}{ auto_concep } & Coeficiente de correlación & $0.495^{* * *}$ & 1.000 \\
& & Sig. (bilateral) & 0.000 &. \\
& $\mathrm{~N}$ & 164 & 164 \\
\hline \hline
\end{tabular}

**. La correlación es significativa al nivel 0.01 (bilateral).

\section{Interpretación:}

En la tabla 1, se observa que el coeficiente de correlación rho de Spearman existente entre las variables muestran una correlación positiva y estadísticamente significativa $\left(r_{s}=.495^{* *}, p\right.$ valor $=0.000<.05)$. Por tanto, se rechaza

la hipótesis nula y se concluye que existe una correlación moderada entre el clima social del aula y el autoconcepto en los estudiantes del nivel secundario de la Institución Educativa $\mathrm{N}^{\circ}$ 2040-Comas.

Tabla 2: Variable clima social y autoconcepto académico/intelectual

\section{Correlaciones}

\begin{tabular}{lllcl}
\hline \hline & & clima_social & intelec_d \\
\hline \multirow{4}{*}{ Rho de Spearman } & \multirow{2}{*}{ clima_social } & Coeficiente de correlación & 1.000 & $0.296^{* *}$ \\
& & Sig. (bilateral) & & 0.000 \\
& & $\mathrm{~N}$ & 164 & 164 \\
& \multirow{3}{*}{ intelec_d } & Coeficiente de correlación & $0.296^{* *}$ & 1.000 \\
& & Sig. (bilateral) & 0.000 & $\cdot$ \\
& & $\mathrm{N}$ & 164 & 164 \\
\hline \hline
\end{tabular}

**. La correlación es significativa al nivel 0.01 (bilateral). 
En la tabla 2 sobre el Clima social y el autoconcepto académico/ Intelectual, está relacionado directamente en los estudiantes del 4to y 5to de secundaria en la Institución Educativa $\mathrm{N}^{\circ} 2040$-Comas, según la correlación de Spearman de 0.296, representando ésta una baja asociación significativa de las variables.

Tabla 3: Variable clima social y autoconcepto social

\section{Correlaciones}

\begin{tabular}{lllll}
\hline \hline & & clima_social & social_d \\
\hline \multirow{4}{*}{ Rho de Spearman } & \multirow{3}{*}{ clima_social } & Coeficiente de correlación & 1.000 & $0.434^{* * *}$ \\
& & Sig. (bilateral) & & 0.000 \\
& & $\mathrm{~N}$ & 164 & 164 \\
& \multirow{2}{*}{ social_d } & Coeficiente de correlación & $0.434^{* *}$ & 1.000 \\
& & Sig. (bilateral) & 0.000 & $\cdot$ \\
& & $\mathrm{N}$ & 164 & 164 \\
\hline \hline
\end{tabular}

**. La correlación es significativa al nivel 0.01 (bilateral).

En la tabla 3 sobre el clima social y el autoconcepto social, está relacionado directamente en los estudiantes del 4to y 5to de secundaria en la Institución Educativa
$N^{\circ}$ 2040-Comas según la correlación de Spearman de 0.434 representando esta una modera asociación de las variables y siendo significativo.

Tabla 4: Variable clima social y autoconcepto emocional/control

\section{Correlaciones}

\begin{tabular}{lllll}
\hline \hline & & clima_social & control_d \\
\hline \multirow{4}{*}{ Rho de Spearman } & Clima_social & Coeficiente de correlación & 1.000 & $0.408^{* * *}$ \\
& & Sig. (bilateral) &. & 0.000 \\
& & $\mathrm{~N}$ & 164 & 164 \\
& \multirow{2}{*}{ control_d } & Coeficiente de correlación & $0.408^{* * *}$ & 1.000 \\
& & Sig. (bilateral) & 0.000 & $\cdot$ \\
& & $\mathrm{N}$ & 164 & 164 \\
\hline \hline
\end{tabular}

**. La correlación es significativa al nivel 0.01 (bilateral).

Como se muestra en la tabla 4 el Clima social y el Autoconcepto emocional/control está relacionado directamente en los estudiantes del 4to y 5 to de secundaria en la Institución

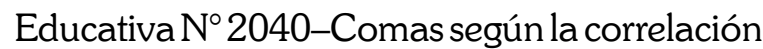
de Spearman de 0.408 representando ésta una modera asociación de las variables siendo significativo.

Tabla 5: Variable clima social y autoconcepto familiar

\section{Correlaciones}

\begin{tabular}{lllll}
\hline & & clima_social & familiar_d \\
\hline \multirow{4}{*}{ Rho de Spearman } & \multirow{3}{*}{ clima_social } & Coeficiente de correlación & 1,000 & $0.366^{* * *}$ \\
& & Sig. (bilateral) & $\cdot$ & 0.000 \\
& & $\mathrm{~N}$ & 164 & 164 \\
& & Coeficiente de correlación & $0.366^{* *}$ & 1.000 \\
& \multirow{2}{*}{ familiar_d } & Sig. (bilateral) & 0.000 & $\cdot$ \\
& & $\mathrm{N}$ & 164 & 164 \\
\hline \hline
\end{tabular}

**. La correlación es significativa al nivel 0.01 (bilateral). 
Como se muestra en la tabla 5 sobre el Clima social y el Autoconcepto en la dimensión familiar está relacionada directamente en los estudiantes del 4to y 5to de secundaria en la Institución Educativa $\mathrm{N}^{\circ}$ 2040-Comas, según la correlación de Spearman de 0.408 representando ésta una modera asociación de las variables. Por el cual, se acepta la relación entre la Clima social y Autoconcepto familiar.

Tabla 6: Variable Clima social y Autoconcepto físico

\section{Correlaciones}

\begin{tabular}{lllll}
\hline \hline & & clima_social & fisico_d \\
\hline \multirow{4}{*}{ Rho de Spearman } & Coeficiente de correlación & 1.000 & $0.279^{* *}$ \\
& \multirow{2}{*}{ clima_social } & Sig. (bilateral) &. & 0.000 \\
& & $\mathrm{~N}$ & 164 & 164 \\
& \multirow{2}{*}{ fisico_d } & Coeficiente de correlación & $0.279^{* *}$ & 1.000 \\
& & Sig. (bilateral) & 0.000 &. \\
& $\mathrm{~N}$ & 164 & 164 \\
\hline \hline
\end{tabular}

**. La correlación es significativa al nivel 0.01 (bilateral).

En la tabla 6 del Clima social y el Autoconcepto en su dimensión físico está relacionada directamente en los estudiantes del 4to y 5to de secundaria en la Institución Educativa $\mathrm{N}^{\circ}$ 2040Re-Comas, según la correlación de Spearman de 0.279 representando esta una baja correlación significativa de las variables.

\section{5.-DISCUSIÓN Y CONCLUSIONES}

De acuerdo a los resultados obtenidos nos muestran que existe una relación entre el clima social del aula y el autoconcepto donde el coeficiente de correlación rho de Spearman da como resultado $r_{s}=0.495^{* *}$, y un $p$ valor $=$ $0.000<0.05$, siendo una correlación positiva y estadísticamente significativa, aunque baja. Este resultado concuerda con lo investigado por Estela (2013) quien en sus resultados llega a la misma conclusión de una correlación positiva. De hecho que el ambiente de aula repercute en los estudiantes porque pasan la mayor parte de tiempo de sus estudios en la institución educativa y el concepto de sí mismos y su imagen se verá reforzado positiva o negativamente si sienten que este sea o no un lugar seguro para su persona. No se descarta tampoco otros factores que afecten estas variables en especial el clima del aula como la disciplina de la institución educativa, la personalidad del estudiante que ha creado tolerancia al medio, características físicas del aula etc. (Sánchez, 2009). Por el cual, hacen que estos resultados sean moderados. Pero lo que es seguro que el clima de aula tiene un impacto en el autoconcepto del estudiante.

En cuanto a la hipótesis específica del clima social y el autoconcepto académico/intelectual está relacionado directamente que según la correlación de Spearman es de 0.296, representando una correlación positiva $y$ estadísticamente significativa, aunque baja de estas variables. Según los resultados existe un $33.5 \%$ que se considera que existe la percepción de un moderado clima social del aula que favorece su trabajo académico o intelectual. Este resultado bajo coincide con el estudio de Meza (2010) en el cual señala que existen otros factores en el clima del aula comola autonomía, cooperación y comunicación, etc. que inciden en el ambiente que el estudiante vive la experiencia de aprendizaje. En los estudios sobre autoconcepto e inteligencia en una de sus expresiones como es inteligencia emocional se señala una correlación positiva (Matalinares, 2005; Amezcua, 2000). Asimismo, el que haya resultado bajo en estas correlaciones puede deberse a que el autoconcepto tiene, por una parte, un papel mediador en el proceso de aprendizaje; $y$, por otra, la existencia de otros factores personales como hábitos de estudio, autoestima positiva, motivación o cómo este se involucre en los estudios dentro del ambiente escolar que hacen que el estudiante tenga un concepto favorable de su capacidad intelectual, como lo señaló Gonzales (1999). 
De acuerdo a los resultados obtenidos del Clima social y el autoconcepto social están relacionados directamente en los estudiantes según la correlación de Spearman de 0.434 representando esta una modera asociación de las variables y siendo significativo. Ello quiere indicar que existe en los estudiantes de la institución educativa un nivel de interacción aceptable entre sus pares y la habilidad para ser aceptados por los otros compañeros de clase. El proporcionar un buen clima en el aula se ve favorecido por programas, talleres, etc. que favorecen aspectos de socialización, de aprendizajes de normas de convivencia para reducir tensiones como señala en sus estudios Sánchez (2009); Sánchez, Rivas y Trianes (2006) y otros que demuestran resultados positivos.

Los resultados obtenidos del Clima social y el autoconcepto emocional/control está relacionado directamente según la correlación de Spearman de 0.408 representando una modera asociación de las variables y siendo significativo. Ello quiere indicar que los estudiantes tienen la percepción de cierto control de la realidad y de las emociones que estas conllevan para desenvolverse en el ámbito escolar de manera acorde o esperada por el grupo de compañeros de clase. Ello ayuda que al percibirse con un manejo adecuado de su conducta y emociones tengan menos riesgos de asumir conductas de riesgo como señala los estudios de Pastor, Balaguer y García (2005).

Los resultados obtenidos sobre el clima social y el autoconcepto en la dimensión familiar están relacionados directamente según la correlación de Spearman de 0.408 representando una moderada asociación de las variables y siendo significativo. Ello demuestra los estudiantes consideran que es importante el relacionarse de forma adecuada con sus familiares. A pesar de estar en la adolescencia donde muchas actitudes son contestatarias frente a la autoridad, los estudiantes consideran importante los nexos familiares. Existe un $28.3 \%$ de estudiantes que consideran positiva la presencia de la familia como un soporte moderado en el ambiente escolar.
Los resultados obtenidos del clima social y el autoconcepto en su dimensión físico están relacionados directamente según la correlación de Spearman de 0.279 representando una baja correlación de las variables. Según Rodríguez (2008), el autoconcepto físico guarda una relación de signo positivo con el bienestar psicológico y una relación negativa con el malestar psicológico. Entonces, un bajo autoconcepto físico en los estudiantes crea una percepción de malestar emocional que arrastra toda la adolescencia. En palabras de Rodríguez la "relación autoconcepto físicobienestar guarda coherencia lógica con la propia naturaleza del bienestar psicológico dado que es un constructo que engloba un juicio acerca de la satisfacción que causa al sujeto determinadas áreas de su vida, entre las que se encuentra la propia persona, es decir, el yo" (2008, p. 158)

Los resultados obtenidos del Clima social y el autoconcepto en la dimensión personal están relacionados directamente según la correlación de Spearman en 0.322, representando una modera asociación de las variables y siendo significativo. De hecho, una percepción positiva de sí mismo lleva consigo a una mejor adaptación al medio que lo rodea dependiendo las condiciones del sujeto como del ambiente escolar. Estudios como los de Ruiz de Azúa et al, 2008; Rodríguez, 2008b, lo confirma.

Finalmente, se ha de buscar sensibilizar a los líderes directivos y docentes de educación para favorecer un clima social escolar que favorezca mayor intercambio de relaciones positivas entre los estudiantes y de los estudiantes con los docentes. Promover en la institución educativa, a partir de la alta dirección, talleres de desarrollo personal, autoconcepto, autoestima y temas afines. Además, promover las posibilidades de crecimiento personal, intelectual, familiar, etc. de los estudiantes. Asimismo, implementar debates y conversatorios entre los docentes y estudiantes. Todo lo cual promueve acciones directas de mejora de la percepción del ambiente escolar y el autoconcepto. 


\section{REFERENCIAS}

American Psychological Association (2010b) Manual de Publicaciones de la American Psychological Association ( $3^{\text {a }}$ ed.). México: Manual Moderno.

Amezcua, J.A. (2000) El autoconcepto y rendimiento escolar en niños de 11 a 14 años. Granada, España: Servicio de publicaciones de la Universidad de Granada.

Arón, A. y Milicic, N. (1999) Clima Social escolar y desarrollo personal. Un programa de mejoramiento. Santiago de Chile: Andrés Bello.

Arón, A. y Milicic, N. (2000) Climas Sociales tóxicos y Clima Sociales Nutritivos para el desarrollo personal en el contexto escolar, en Revista Psikhé, Vol. 9, N. ${ }^{\circ}$ 2, 117-123.

Bass, R. y Del Rey, R. (2003) La violencia escolar. Estrategias de prevención. Barcelona: Graó.

Branden, N. (1993) El poder de la autoestima. Barcelona: Paidos.

Centty, D. (2006) Manual metodológico para el investigador científico. Arequipa: Nuevo Mundo, investigadores y consultores.

Chuquillanqui, I. S. (2012) Funcionamiento familiar y autoconcepto de los alumnos del sexto grado de las instituciones educativas de la red 8 Callao (Tesis de Maestría en Universidad San Ignacio de Loyola, Lima, Perú) Recuperado el 8 de noviembre 2014 de http://goo.gl/Tp8yNB.

De Narváez, M ${ }^{\mathrm{a}}$ T. (2002) Aprendiendo y creciendo juntos. Vol. III. Editora contusalud.com. Recuperado el 16 de junio de 2004 de la wold wide web: www. contusalud.com/wedside/fólder/ sepa_psicología-autoestima.htm

Esnaola, I.; Goñi, A. y Madariaga, J. (2008) El autoconcepto: perspectiva de investigación, en Psicodidáctica. Vol. 13, No. 1, 179-194.

Epstein, N. (1990) Identificación de los factores educativos de un modelo de resiliencia que los padres estén activamente involucrados. Recuperado el 11 de febrero de 2010 www.human.ula.ve/ doctorado en educación/ documentos/ anzola. Pdf

Estela, F. (2013) Clima social escolar y autoconcepto en los estudiantes del IV ciclo de educación primaria de la I.E N¹23 "los árboles", Santa Anita-2013-Tesis de Maestría. Lima: Universidad César Vallejo.

Fariña, F; García, P. y Vilariño, M. (2010) Autoconcepto y procesos de atribución: estudio de los efectos de protección/riesgo frente al comportamiento antisocial y delictivo, en la reincidencia delictiva y en el tramo de responsabilidad penal de los menores. Revista de Investigación en Educación, No 7, 2010, España: Universidad de Santiago de Compostela, 113-121.

García, F. y Musitu, G. (2009) AF5: Autoconcepto Forma 5 ŚAF5: Self-concept form5Ș (3 ed.). Madrid, España: Tea.

Gonzales, M. (1999) Autoconcepto y rendimiento escolar. Universidad de Navarra: EUNSA.

Gonzales, O. (2011) La presión sociocultural percibida sobre el autoconcepto físico: Naturaleza, medida y viabilidad. Tesis doctoral. Universidad del País Vasco Euskal Herrico Unibertsitateko. España: Servicio Editorial de la Universidad del País Vasco. 
Harter, S. (1999) The construction of the self. A developmental perspective. New York: The Guilford Press.

Hernández, R., Fernández, C. y Baptista, M. (2010) Metodología de la Investigación. (5. a ed.). México: Mc Graw - Hill.

Loperena, M. (2008) El autoconcepto en niños de cuatro a seis años, en Tiempo de Educar, Vol. $9, \mathrm{~N}^{\circ} 18,307-327$.

Matalinares et al (2005) Inteligencia emocional y autoconcepto en Colegiales de Lima metropolitana, en Revista IIPSI. Vol. 8, N. ${ }^{\circ} 2$ - 2005. Lima: Universidad Mayor de San Marcos. 41 - 55.

Markus, H. y Kunda, Z. (1986) Stability and malleability of the self-concept, en Journal of personality and social psychology, Vol. $51, \mathrm{~N}^{\circ}{ }^{\circ} 4,858-866$.

Meza, C. (2010) Clima de aula y rendimiento académico en estudiantes de cuarto y quinto grado de secundaria en una institución educativa del Callao (Tesis de maestría) Lima: Universidad San Ignacio de Loyola.

Moos, R. (1979) Evaluating educational environments. S. Francisco: Jossey.

Moos, R. H., Moos, B. S. \& Tricket, E. J. (1989) Escalas de clima social: Familia, Trabajo, Instituciones Penitenciarias, Centro Escolar. Madrid: Consulting Psychologists Press. Adaptación española, TEA Ediciones S.A.

Peralta, F.J. y Sánchez, M.D. (2006) Relación de autoconcepto y el rendimiento académico en alumnos de educación primaria. Revista electrónica de investigación Psicoeducativa y Psicopedagógica. I (I), 95-120. ISSN:1696-2095.

Pastor, Y.; Balaguer, I. y García-Merita, M. (2005) Relaciones entre el autoconcepto y el estilo de vida saludable en la adolescencia media: un modelo exploratorio, en Psicothema 2006. Vol. 18, N. ${ }^{\circ} 1,18-24$. CODEN PSOTEG.

Sánchez, C. (2009) Nivel de implicancia en bullying entre escolares de educación primaria. Relación con el estatus social y la percepción del clima social, familiar y escolar. (Tesis doctoral). Universidad de Murcia. España: Murcia.

Sánchez, A. M.; Rivas, M.T. y Trianes, M.V. (2006) Eficacia de un programa de intervención para la mejora del clima escolar algunos resultados Revista electrónica de investigación Psicoeducativa. Vol. 4 (2), $\mathrm{N}^{\circ}$ 9, 353-370.

Peralta, F. J. y Sánchez, M. D. (2006) Relación de autoconcepto y el rendimiento académico, en alumnos de educación de primaria. Revista electrónica de investigación Psicoeducativa y Psicopedagógica. I (I),95-120.

Rodríguez, A. (2008) Autoconcepto físico y bienestar/ malestar. Revista de Psicodidáctica, Vol. 14, N. ${ }^{\circ}$ 1, 2009, 155-158, España: Universidad delPaís Vasco/Euskal Herriko Unibertsitatea.

Rodríguez, A. (2008b) Malestar psicológico y el autoconcepto físico. En A. Goñi (Ed.). El autoconcepto físico: Psicología y educación. Madrid: Pirámide. 
Ruiz de Azua, S., Goñi, A. y Madariaga, J. (2008) Variabilidad del autoconcepto físico. En A. Goñi (Ed.), El autoconcepto físico: Psicología y educación. Madrid: Pirámide.

Trianes, M. et al (2006) Un cuestionario para evaluar el clima social escolar. En Psicotema Vol. 18. N. ${ }^{\circ}$ 2, pp. 272-277. España: Universidad de Málaga. Recuperado en:

http://www.psicothema.com/pdf/3209.pdf.

Valderrama, S. (2013) Pasos para elaborar proyectos de investigación científica. Lima: San Marcos.

Valdez, A. A. y Carlos, E.A. (2014) Relación entre el autoconcepto social, el clima familiar y el clima escolar con el bullying en estudiantes de secundaria. en Revista Avances en Psicología Latinoamericana/Bogotá (Colombia) Vol. 32 N. ${ }^{\circ}$ 3. 447-457.

Fecha de recepción: 18-03- 16

Fecha de aceptación: 13-06-16 